\title{
Towards Paradigm Change of Formative Assessment in the Undergraduate Curriculum: The Participatory Action Research (PAR)
}

\author{
Sylvia Mustika Sari ${ }^{1, *}$, Iis Inayati ${ }^{1}$, Atia Sidiqa ${ }^{2}$ \\ 'Medical Faculty Universitas Jenderal A. Yani \\ 2Dental Faculty Universitas Jenderal A. Yani \\ ·Corresponding author. Email: sylvia.mustikasari@lecture.unjani.ac.id
}

\begin{abstract}
The concept of "assessment for learning" has been widely defined as a form of assessment that facilitates the improvement of students' learning. However, there's still limited evidence of real implementation of formative assessment, especially related to the sociocultural issues. This study explored the formative paradigm change in students and lecturers in our context. A participatory action research approach was used in two groups of the undergraduate medical teachers $(\mathrm{N}=18)$, and students $(\mathrm{N}=30)$. This research contains two cyclical processes of reflection for over 4 months. In between the cycle, we have workshops and continuing discussions about formative assessment. The FGD and in-depth interviews were done to explore the gap between the cycle qualitatively. The results showed the gaps and challenges in changing the paradigm. We identified three themes of paradigm changes : (1) "Formative is the assessment of knowledge" to "the assessment of thinking skills and professionalism"; (2) Feedback "derived in group and one direction explanation" to "individually driven and a dialogic form"; (3) Formative assessment result is "score-minded" to "the improvement of thinking skills and process-minded". Besides, we found the themes of challenges in these paradigm changes, such as (1) the good practice in "how to" assess and feedback dialog in cultural context; (2) students' self-reflection skills ; (3) student and teachers' collaboration. We conclude that there is no simple way to implement the new paradigm of assessment, especially related to the cultural adaptation. We recommend this participatory approach to be a crucial part in the assessment paradigm changes.
\end{abstract}

Keywords: "Formative", "undergraduate", "participatory action research".

\section{INTRODUCTION}

Currently, paradigm in student assessment in medical education has a new direction to the exploration of learning impact in student assessment or the concept of "assessment for learning" [1] [2]. The consideration of the impact on student learning itself not a new concept, since the phrase "assessment drives learning" has been pronounced by Miller many decades ago. However, the mechanism on the relationship between assessment and student learning still needs to explore [2]. Some researches believe that the mechanism to improve the quality of student learning in undergraduate medical education is a continuous constructive feedback during formative assessment [1] [3]. The development of learning impact in formative assessment will answer the problems that often occur when the results of the summative assessment are used as indicators of success by students or even the faculty [4].

Considering on the mechanism of "assessment for learning" in undergraduate medical education, we highlighted the important aspect during the adaptation such as continuous feedback in formative assessment. Lersen et al [5] and Green et al [6] introduced a concept of "test enhance learning" which is defined as a measurement that is carried out periodically and is followed by feedback. This is supported by various studies which prove that this concept results in better learning quality and retention in students. It is also expressed by Gibbs and Simpson [7] that the impact of the assessment system on the quality of student learning is influenced by the quality and quantity of feedback.

This underlies the mechanism of learning impact or "assessment for learning" concept needs a strong 
relationship between undergraduate student and teacher, which is related to the cultural factor. The cultural aspect found to be influenced the process of providing feedback. [8]. Suhoyo, et al [8] distinguished the Indonesian and Dutch students which had differences, especially in the power-distance approach. In Indonesia, students tend to have low individuality, making it difficult to change their behavior autonomously, and the approach is that there is a "power distance" between lecturers and students which will affect the giving of feedback. Furthermore, the experience of receiving feedback will have an influence on student learning motivation [9].

In undergraduate learning at the Medical Faculty of Unjani, formative assessments are carried out during the tutorial process, clinical skills training and other learning methods. One of the challenges in developing formative assessments is that the implementation of formative exams currently does not have an optimal role based on the assessment results data provided by the lecturers. The results tend to be the same and students have a tendency to prepare for summative exams in a short amount of time.

In our previous research through a phenomenological qualitative approach, we found that students and lecturers in our faculty perceived the formative assessment (1) as a "score-minded", (2) as the assessment of knowledge, and (3) contains feedback that derived in group as one direction explanation [10].

Based on our preliminary study, we found the big challenges to adopt the concept of "assessment for learning" in our context especially related to our cultural factor such as a hierarchical relationship between students and lectures. Therefore, we develop our research questions as follows:

1) How is the paradigm change in students and lectures at our undergraduate medical education about formative assessment that drives learning?

2) What is the possible challenges in the implementation of formative assessment that drives learning?

\section{METHODS}

\subsection{Study design}

This study uses a Participatory Action Research (PAR) approach to develop a formative assessment model at the undergraduate stage. PAR design is used as the main step in the development of a model that is influenced by sociocultural factors. Through this design, researchers involve participants from the beginning of the modeling process to the implementation, observation and reflection of the model.

\subsection{Respondents}

The population of this study were students and lecturers at the undergraduate level in the Medical and Dentistry Faculty of Unjani. Respondents was carried out purposively, namely 30 students, as well as 18 medical lecturers from Medical and Dentistry Faculty.

\subsection{The steps of PAR cycle}

The Participatory Action Research (PAR) in this study contains a continued two cycles of action-reflection, which adapted from Mash B, Meulenberg-Buskens [11]. In the first cycle, we developed the workshop for medical and dentistry teachers about the formative assessment such as how to give a constructive feedback and the continuing formative assessment. After the first workshops, teachers started to continue the learning process with the formative assessment. This cycle ended by our first reflection in focus group discussion about the formative assessment experience from teachers and students.

In the second cycle, we developed the workshops about formative assessment for students and followed by continued discussion on WhatsApp Group of teachers and students. Our second cycle ended with second reflection in focus group discussion about the formative assessment experience from teachers and students. Figure 1 represents our PAR cycle.

\subsection{Data analysis}

The qualitative data from two reflection-phase in PAR cycle analyzed by qualitative data inquiry with thematic analysis based on our preliminary study. The thematic framework used such as (1) The definition of formative assessment; (2) Providing feedback in formative assessment; (3) The challenging factor to implement the new paradigm. 


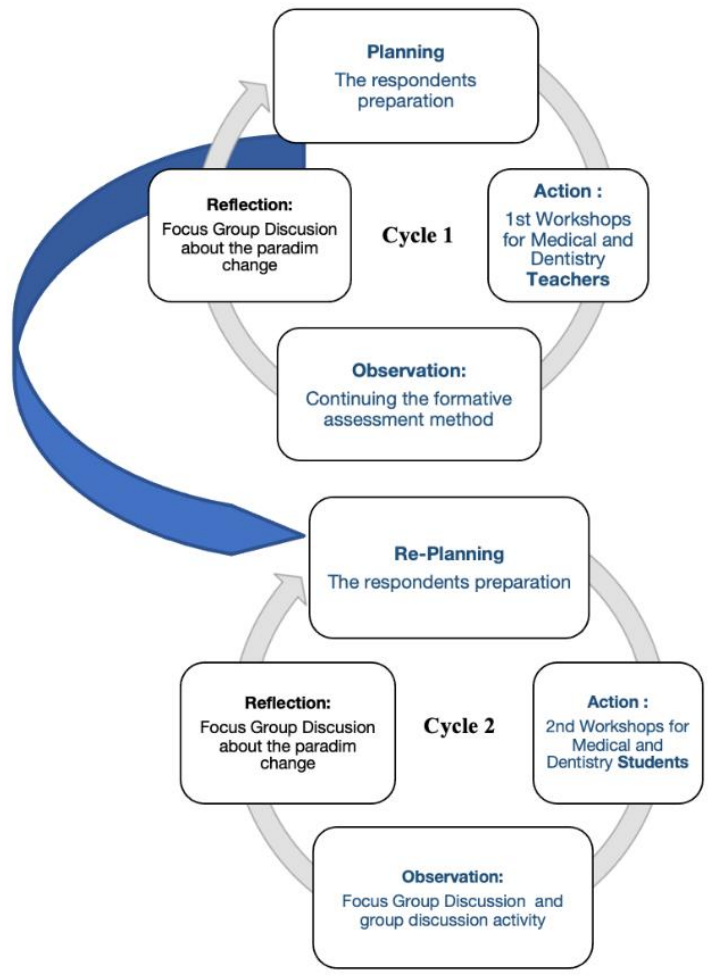

Figure 1. The Adopted PAR Cycles

\section{RESULTS}

In this study, two reflection cycles were carried out in two groups of lecturers and students. Between the two reflection cycles, workshops and discussions were held regarding the possibility of changing the mindset of both lecturers and students. A paradigm shift in formative assessment also requires a change in learning patterns. This is because in the formative assessment which is in accordance with the concept of "assessment for learning", it contains student learning skills to use lecturer feedback as the basis for improving the quality of their learning. Through two cycles of reflection on the expected changes in the formative assessment at the undergraduate stage of medicine and dentistry, a paradigm shift was found which can be described as themes: (1) The scope of formative assessment for knowledge acquisition becomes an assessment of students' professional mindset and behavior; (2) Providing feedback containing learning material in one direction into a form of dialogue about individual abilities on a regular basis; (3) Formative focus on the final score becomes an approach to advancing the mindset and professional behavior in the learning process. In the sections below, the acquisition of each theme will be explained through deductive logic.

\subsection{Change in formative assessment definition}

In the first reflection cycle, the theme was obtained that the formative assessment according to lecturers and students was an assessment of mastery of knowledge. This is based on the existence of quotations related to the scope of the formative assessment which is analogous to the pre and post test. Based on the results of the reflection of the first cycle, lecturers and students put forward scores and get formative assessment feedback as "daily scores" which are components of the final score. Some of the quotations from students also described a sense of injustice when they got the results of these assessments.

The quotes from lecturers and students that reinforces this theme are:

"In the laboratory session, it is clearer because there is a pre test and post test to determine the formative value" (Med Teacher_KU_12)

"If we answer incorrectly it means the score is not good" (Med Student_KU_03)

The scope of formative assessment in the first cycle was dominated by perceptions of assessment of knowledge through pre and post tests, student ability to answer questions and assessment of student activeness during discussion. Furthermore, in the second cycle, there were perceptions of lecturers and students that led to the understanding that the formative assessment process should contain an assessment of students' thinking patterns and professional behavior during learning.

"The assessment is whether the students is perform domination in discussion or not ... sometimes I think, if the student dominate in discussion, but the answer is correct, what is the value?" (Med Teacher_KG_02)

\subsection{Orientation change in formative assessment}

In the first cycle of FGD, it was found that there was a tendency that the formative assessment paradigm of both lecturers and students was result oriented. In the regulation of the learning system, there is the term daily value which is representative of formative assessment. However, this is often considered incompatible with student performance. This phenomenon is found in the data of lecturers and students. The following quotes are able to describe it.

"The formative is determined by the percentage to the final value" (Med Teacher_KU_08)

"What is confusing determines the score" ((Med Teacher KU_02) 
Based on the quotations from the lecturers, it was found that there was a tendency to lack of standardization from the lecturers in conducting assessments. This has an impact on the mindset of students about the formative assessment.

"Actually, I wanna ask what is being assessed huh?" (Med Student_KU_02)

"For me to be judged or not, there is no effect." (Dentistry Student_KG_08)

In the workshop intervention and discussions before running the second cycle, students and lecturers have gained the meaning and function of formative assessment.

"We should be able to periodically assess students. (DKU_02)

"We have to see the progress" "(DKU_02)

"We want an appreciation of our ability in learning" (MKU_02)

\subsection{Change in providing feedback on formative assessment}

In the initial cycle, we found that the definition of feedback that is felt by both lecturers and students is an explanation from the lecturer about the learning material that is delivered at the end of the discussion session. Not many lecturers provide feedback regarding students' mindset abilities or professional behavior.

"For me, the meaning of feedback is the answer from the lecturer, as usually after the discussion the students ask "what is the feedback, Doc?" ... Then I explain the answer" (Med Teacher_KU_18)

In the second cycle, there was a change in perception that underlies the paradigm shift of students and lecturers which is completely summarized in Figure 2 and 3 .

"I think feedback has to be individual, but it is going to be a long time of tutorial..hehe" (Med Student_KU_09)

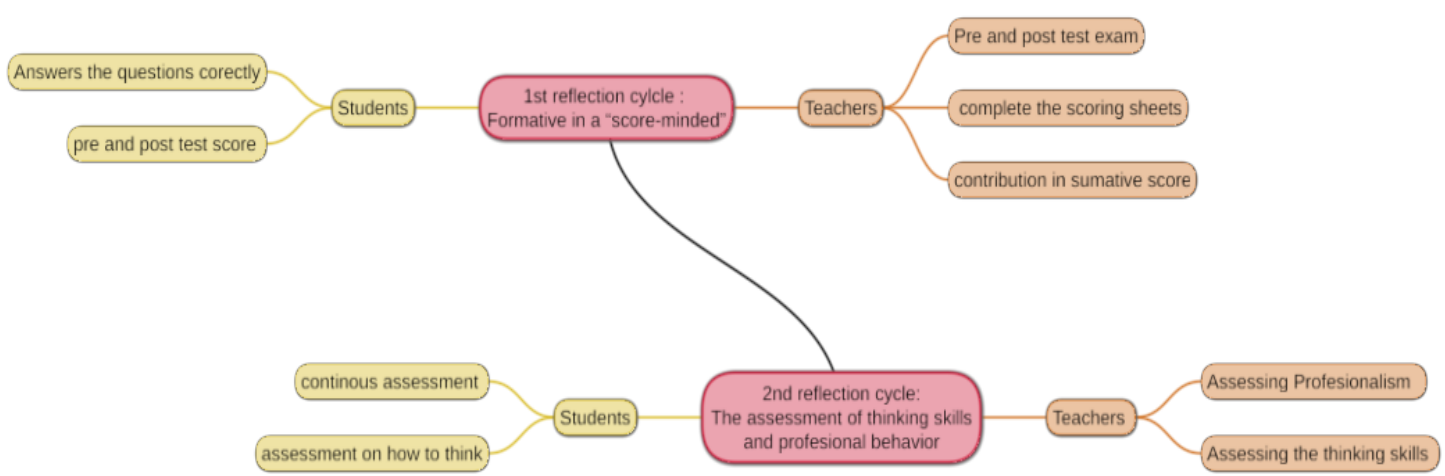

Figure 2. The paradigm changes of formative assessment definition

\subsection{The challenges}

In the second reflection cycle, we found the themes that represent the challenges in these paradigm changes, such as (1) the good practice in "how to" assess and feedback dialog in cultural context; (2) students' selfreflection skills ; (3) student and teachers' collaboration. The quotes from lecturers and students that reinforces this theme are:

"Actually, I don't know how to introduce the dialog with our teacher, I feel somehow .. huum.. afraid?" (Med_Student_KU_07)

"What if they just keep silence?.." (Dentistry_Teacher_KG_02)
An ideal feedback represents the interaction between lecturer and student that will affect social relations between lecturer and student. In the context of hierarchical culture with high power-distance, lecturers are in a higher position than students. The themes found in this study showed that impression. In that way, feedback communication form is one of the biggest challenge during formative assessment, related to student and teachers collaboration. Besides, from teachers' point of view, we found the impression of the lack in students' reflection skills. 


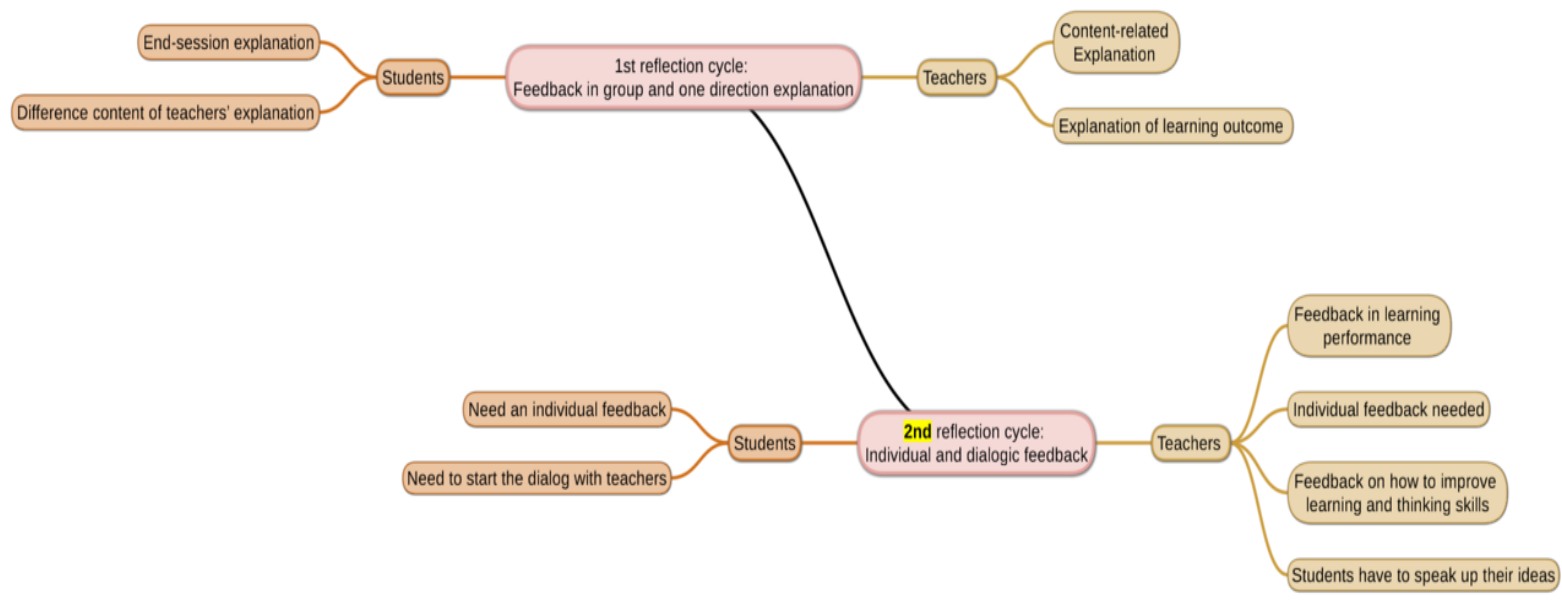

Figure 3. The paradigm changes on providing

\section{DISCUSSION}

A paradigm shift in the assessment method in medical education that has an impact on improving the quality of student learning is not an easy pathway. Previous teaching patterns and culture influenced the adaptation results of various concepts that were first developed in different cultural contexts. This phenomenon is the background of the use of Participatory Action Research (PAR), which is an action research method by promoting the active participation of the respondents. In the first reflection cycle, the theme was obtained that the formative assessment according to lecturers and students was an assessment of mastery of knowledge. This is based on the existence of quotations

Providing feedback is an important aspect as a driving force in formative assessment. Through feedback, lecturers are expected to be able to provide an overview of the position of students in achieving their learning knowledge and skills. Furthermore, students are expected to be able to make this feedback as the basis for their self-development. Feedback is a fundamental aspect of formative assessment. Through constructive feedback, students are able to reflect on their current abilities which are the basis for improving the quality of their learning. This is in accordance with the rules of "assessment for learning". Research related to giving feedback currently leads to a form of "dialogue" which not only prioritizes the skills of lecturers in providing feedback and the ability of students to receive feedback, but is directed at the quality of interaction in the feedback process in the form of dialogue. Ideal feedback is input from lecturers who are closely related to objective observation results, related to the scope of the formative assessment which analogous to the pre and post test. The exam format can be used to see the student's ability in mastering the material or what is known as "Test Enhaced Learning" (TEL). Larsen et al [5] stated that this can be meaningful if it is followed by direct feedback so that students are able to evaluate themselves.Based on our findings, the lecturer has gained insight into the essence of formative assessment which is oriented towards improving the quality of student learning during the learning process. This is supported by students who also have the desire to be assessed in the form of appreciation related to improving their abilities

\section{CONCLUSION}

Through two cycles of reflection in this study, a paradigm shift in medical and dentistry student and teachers were found to be at a "starting point" should be continue to a bigger number of students and teacher. We conclude that there is no simple way to implement the new paradigm of assessment, especially related to the cultural adaptation. We recommend this participatory approach to be a crucial part in the assessment paradigm changes. Future research can use the results of this study as a basis for developing a formative assessment model at the undergraduate stage in the context of Indonesian culture. 


\section{ACKNOWLEDGMENTS}

This research founded by LPPM Universitas Jenderal Achmad Yani.

\section{REFERENCES}

[1] Schuwirth, L. W. T., \& van der Vleuten, C. P. M. (2018). How 'Testing' Has Become 'Programmatic Assessment for Learning.' Health Professions Education. https://doi.org/10.1016/J.HPE.2018.06.005

[2] Cilliers, F. J. (2015). Is assessment good for learning or learning good for assessment? A. Both? B. Neither? C. It depends? Perspectives on Medical Education, 4(6), 280-281. https://doi.org/10.1007/s40037-015-0229-1

[3] Wiliam D. What is assessment for learning? Studies in Educational Evaluation. Elsevier . 2011; 37: 314 Available from: www.Elsevier.com/studedu.

[4] Kulasegaram _K \& Rangachari PK. Beyond "formative" assessments to enrich student learning. Advanced Physiology Education Journal 2017; 42: 5-14, 2018; doi:10.1152/advan.00122

[5] Larsen DP, Butler AW \& Roediger HL. Test enhance learning in medical education. Medical Education 2008; 42: 959-966 doi:10.1111/j.13652923.2008.03124.X

[6] Green ML, Jeremy J. Moeller \& Spak JM. Testenhanced learning in health professions education: A systematic review: BEME Guide No. 48, Medical Teacher, 2018; 40:4, 337-350, DOI: 10.1080/0142159X.2018.1430354

[7] Gibbs G, Simpson C. Conditions under which assessment suports students learning. Learning and teaching in higher education 2005: p.16-20.

[8] Suhoyo Y, Van Hell EA, Prihatiningsih TS, Kuks JBM \& Schotanus JC. Exploring cultural differences in feedback processes and perceived instructiveness during clerkships: Replicating a Dutch study in Indonesia. Medical Teacher 2014; 36: $223-229$

[9] Sari SM. How does feedback in clinical teaching motivate student's learning?. Proceeding $5^{\text {th }}$ SEARAME International Conference 2018. Available from: http://symposium.fk.ugm.ac.id/jit/wpcontent/uploads/2019/05/PROCEEDING-5THSEARAME-INTERNATIONALCONFERENCE-2018-min.pdf

[10] Sari SM. The Perceived of Students and Teacher of Formative Assessment in Undergraduate Medical Education: A Qualitative Study. Conference HandBook.
Asia Pasific Medical Education Conference 2020. Available from: http://medicine.nus.edu.sg/cenmed/apmec17/ documents/APMEC Conference\%20Handbo ok.pdf

[11] Mash B, Meulenberg-Buskens I. 'Holding it lightly': the co-operative inquiry group: a method for developing educational materials. Med Educ. 2001; 35(12):1108-14.

[12] Ramani, S., Könings, K. D., Ginsburg, S., \& van der Vleuten, C. P. M. (2019). Twelve tips to promote a feedback culture with a growth mind-set: Swinging the feedback pendulum from recipes to relationships. Medical Teacher, 41(6), 625-631. https://doi.org/10.1080/0142159X.2018.1432850 